\title{
For Whom the Bell Does Not Toll The invisible saga of cancer caregivers
}

Ritu Lakhtakia ${ }^{1}$ and "Ikram Burney ${ }^{2,3}$

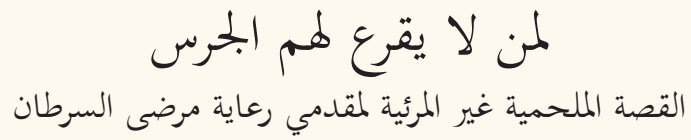

ريتو لكتاكيا و إكرام بيرني

$\mathrm{C}$ NCER CARE CAN bE SEEN as a threelegged stool. Whereas, the centre of attention is the patient, care cannot be provided without healthcare providers and caregivers. Quality caregiving enhances the patient's physical and emotional well-being. The informal caregivers in oncologic practice need enhanced support, whether faced with the imminent demise of a cancer patient or in the long-term care of survivors. Yet, in cancer care protocols, informal caregivers are often neglected; a compromise of their quality of life is a foregone conclusion. ${ }^{1}$ For example, a physician whose sibling is wasting away from advanced cancer may express their angst by expressing "she [the cancer patient] is the strong one in the family". Young parents may mask their emotions to hide their distress of seeing their child with neuroblastoma buoyant between cycles of chemotherapy.

What do cancer caregivers witness, cope with, buffer and express? A spouse, parent, sibling, partner or close friend may take the first steps to becoming a caregiver when they are present when the diagnosis is given. The impact of this news is, at best, a close second in intensity, to what the patient experiences. One has witnessed both sides of the coin - the patient who receives the diagnosis with equanimity as those close to the patient dissolve in tears or, a family member assuming strength and hope when the patient retreats into shock or denial. Caregiver's involvement in cancer care varies from culture to culture, but also across time in care of the same patient. The caregiving spectrum can range from silent/supportive to paternalistic/decision-maker.

As the initial trauma of acceptance of the diagnosis subsides, caregivers begin a journey of frequent hospital visits and witnessing the physical and psychological effects of therapy. Many undiscovered attributes of the caregiver can arise: patience, attentiveness, dependability and trustworthiness. A absence from the workplace, lack of attention to daily chores and children's needs and abandonment of lifestyle activities may become the new norm for the caregiver. There may be simultaneous financial struggle due to mounting medical expenses. In addition, legal ramifications of inheritance, in the event of the patient's death, become an unspoken dilemma. Quality of life, including effect on physical health, spirituality, psychological distress and social support has recently been reviewed across a range of caregivers. ${ }^{2}$

The success of advanced oncologic therapy with resultant long-term survival has brought hope to patients and families. Cancers are curable or can be treated as chronic diseases. The effort to sustain chronic caregiving increasingly affects the caregiver's personality and physical well-being. As the disease progresses, the caregiver may have to take responsibility for adopting unproven or minimally beneficial and often expensive and confusing second- and third-line options. Homecare in the palliative setting may challenge the caregiver by the administration of pain relief or other medications as well as a more intimate observation of deterioration and suffering. The Stressful Caregiving Adult Reactions to Experiences of Dying scale has been used to aid in early identification of caregivers stress level due to dying patients' critical health events. ${ }^{3}$

When cancers progress rapidly, attempts at coping with the situation either induce yeoman efforts or prove overwhelming; witnessing a child's suffering can be particularly devastating. Post-bereavement, caregivers are called cancer survivors and their recovery can manifest in multiple ways: a lifelong phobia of cancer; failure to return to a healthy lifestyle or relationships; continued support to other family members such as children and parents; and/or long-term economic burden.

Despite the many challenges integral to cancer care, some caregivers may gain a sense of satisfaction in this humanitarian mission; caregiving may enrich 
life and instil pride and a sense of purpose. In some cultures, such as in the East, familial bonds oblige one to provide care to other family members, providing a sense of satisfaction and dutifulness. Religious anchors endorse caregiving as a noble means of obeying the commands of God. Some caregivers see the opportunity to learn new skills, socialise, make friends and have gone on to champion this cause, such as by creating non-governmental organisations.

The emergence of excellence in oncologic therapies on the one hand and holistic cancer care on the other, have been the hallmarks of the fight against cancer. Out of necessity, research has focused on the patients and their disease while the multi-nuanced experiences of cancer caregivers remains undiscovered and as a result they may be unprepared and untrained to support their loved ones and protect themselves. Psychoeducational interventions have been the predominant approach to caregiver interventions while adaptive flexibility in the format of interventions is recommended. ${ }^{4}$ Further research should be carried out on outpatient cancer care and reinforcement of caregivers' capacity and wellbeing at all levels of healthcare.

Misconceptions of cancer caregivers should be addressed via recognition, awareness and supportive inclusion in the cancer care paradigm. In this era of rapidly evolving and expanding technology such as educational websites, internet-based support groups and smartphone applications, a technology-driven intervention may be helpful in this regard. Healthcare professionals and social support mechanisms should unite and support each other to empower silent caregivers.

\section{References}

1 Kilic ST, Oz F. Family caregivers' involvement in caring with cancer and their quality of life. Asian Pac J Cancer Prev 2019; 20:1735-41. https://doi.org/10.31557/APJCP.2019.20.6.1735.

2 Ochoa CY, Buchanan Lunsford N, Lee Smith J. Impact of informal cancer caregiving across the cancer experience: A systematic literature review of quality of life. Palliat Support Care 2019; 7:1-21. https://doi.org/10.1017/S1478951519000622.

3 Galatsch M, Prigerson HG, Schnepp W, Zu Sayn-Wittgenstein F, $\mathrm{Li}$ J. Caregiver exposure to critical events and distress in home-based palliative care in Germany a cross-sectional study using the Stressful Caregiving Adult Reactions to Experiences of Dying (SCARED) scale. BMC Palliat Care 2019; 18:9. https://doi.org/10.1186/s12904-019-0395-8.

4 Ferrell B, Wittenberg E. A review of family caregiving intervention trials in oncology. CA Cancer J Clin 2017; 67:318-25. https://doi.org/10.3322/caac.21396. 